\title{
Synthesis and Evaluation of Triazolyl Dihydropyrimidines as Potential Anticancer Agents
}

\author{
Rajanna Ajumeera ${ }^{1}$, Ganapathi Thipparapu ${ }^{1}$, Shireesha Boyapati ${ }^{2}$, Bharath Singh Padya ${ }^{1}$ and Vijayalaxmi Venkatesan ${ }^{1}$ \\ ${ }^{1}$ Division of stem Cell Research, National Institute of Nutrition, Tanaka, Hyderabad, Telangana, India \\ ${ }^{2}$ Department of Pharmaceutical Chemistry, Telangana University, Dichpally, Telangana, India \\ Correspondence: Rajanna Ajumeera, Division of stem Cell Research, National Institute of Nutrition, Tanaka, Hyderabad, \\ Telangana, India.
}

Received: September 17, 2018 Accepted: November 1, 2018 Online Published: November 8, 2018

doi:10.5539/ijc.v10n4p18

URL: https://doi.org/10.5539/ijc.v10n4p18

\begin{abstract}
Novel N - triazolyl 3(a-f) and O-triazolyl (4a-f) derivatives of 4, 6-diaryl-1, 4-dihydropyrimidines were synthesized through mannich reaction. All compounds were characterized by physical and spectral data. These compounds were screened for in vitro efficiency in human breast cancer cell (MCF-7\&MDA-MB-231) lines and found to have very good anti-proliferative activity. Among all compounds of $\mathbf{4 b}, \mathbf{3 e}, \mathbf{4 e}$ endowed with lesser respective $\mathrm{IC}_{50}$ values of 31.94, 55.73, 55.03 $\mu \mathrm{M}$ in MCF-7 cells and 41.50, 35.28, 32.06 $\mu \mathrm{M}$ in MDA-MB 231 cells by MTT assay. In further studies, Compounds $\mathbf{4 b}, \mathbf{3 e}, \mathbf{4 e}$ were found to arrest cell growth at S phase in MCF-7 cells. In MDA-MB 231 cells, $\mathbf{4 b}, \mathbf{4 e}$ were found to arrest the cells in $\mathrm{S}$ phase, and compound $3 \mathbf{e}$ found to arrest G2/M phase when compared to the standard drug tamoxifen, arrested S phase in MCF-7 cells and G0/G1 phase in MDA-MB 231 cells.
\end{abstract}

Keywords: dihydropyrimidines, triazoles, anticancer activity, cell cycle, breast cancer cells, MTT assay

\section{Introduction:}

Pyrimidines were nitrogen compounds, discovered in 1893 and are cyclic amines. It is also known as $m$-diazone (or) 1 , 3-diazine and got the recognition as parent nucleus of large group of heterocyclic compounds. The reduced pyrimidines i.e. dihydropyrimidines (DHPM) is also an interesting heterocyclic ring, that was found to possess a vital role in numerous biological processes as it is the core nucleus of the endogenous molecules like nucleic acids, co-enzymes, several vitamins, purines and liposaccharides. It has been reported for various biological activities like antibacterial (Sangaraiah, et al., 2012), antifungal (Rami, et al., 2013), antiviral (Hockova, et al., 2003, Breault, et al., 2003), anticancer (Sosnicki, et al., 2014), antihypertensive (Chikhale, et al., 2009) with calcium channel blocking and dihydrofolate reductase inhibition. In addition, these compounds emerged as potential $\alpha_{1 a}$-adrenergic antagonists (Schneider, et al., 2003), vasodilators (Cernecka, et al., 2012), antiatherosclerotic (Dobrusin, et al., 2001), antidiabetic (Lauro, et al., 2010), antiplatelet aggregation (Bruno, et al., 2001) and neuropeptide antagonists (Chikhale, et al., 2009). The chemistry of Pyrimidines has been extensively studied, since the Pyrimidine is a symmetrical molecule about the line passing through $\mathrm{C}_{2}$ and $\mathrm{C}_{5}$, the $\mathrm{N}_{1}$ and $\mathrm{N}_{3}$ positions are equivalent and so are $\mathrm{C}_{4}$ and $\mathrm{C}_{6}$. When a $-\mathrm{OH}$ or $-\mathrm{NH}_{2}$ group is present at the 2, 4 or 6 position then there is a keto-enol or amino-imino tautomerism existed with oxo and imino forms.

The other nitrogen based heterocyclic nucleus 1,2,4-triazole was associated with diverse range of pharmacological activities such as analgesic, antiinflammatory, antiasthmatic, anticholinergic, antihypersensitive, antibacterial, antifungal and diuretic activity (Birendra et al, 1984, Kothari et al, 1980, Sengupta \& Misra, 1981, Sarmah \& Bahel, 1982). Mannich bases are well known for their medicinal values such as dynamic chemotherapeutic agent (Anticancer, antibacterial, antifungal, anthelmintic, anti-HIV, antiviral, antitubercular \& antimalarial activities), anti-inflammatory, anticonvulsant, analgesic \& antipsychotic activities (Koksal, et al., 2007; Vashishtha, et al., 2004, Sriram, et al., 2009; Mulla, et al., 2011). The biological data of both the heterocycles provoked us to synthesize some new dihydropyrimidine derivatives containing 1,2,4-triazole ring.

In the present investigation, based on the structural significance of dihydropyrimidines and mannich bases, we designed and synthesized mannich bases of dihydropyrimidinone with regioselectivity. Mannich bases were synthesized by reacting DHPR with secondary amine and formaldehyde in the presence and absence of catalytic amount of potassium carbonate to get $\mathrm{O}$ - and N-mannich bases respectively. DHPM were synthesized from chalcones containing different 
substitutions on the both aromatic rings. Different secondary amines were used for the synthesis of mannich bases such as aliphatic, aromatic and heterocyclic amines (Bandgar, et al., 2010; Trivedi, et al., 2008; Shah, et al., 2010; Pandeya, et al., 2000). The synthesized compounds (3a-3f \& 4a-4f) were evaluated for anti-cancer activity in vitro in human breast cancer cell (MCF-7 \& MDA-MB-231) lines. The toxicity of these derivatives was predicted by using in silico methods (Ganapathi et al, 2016).

\section{Synthesis of Dihydropyrimidine Derivatives}

\section{Scheme:}

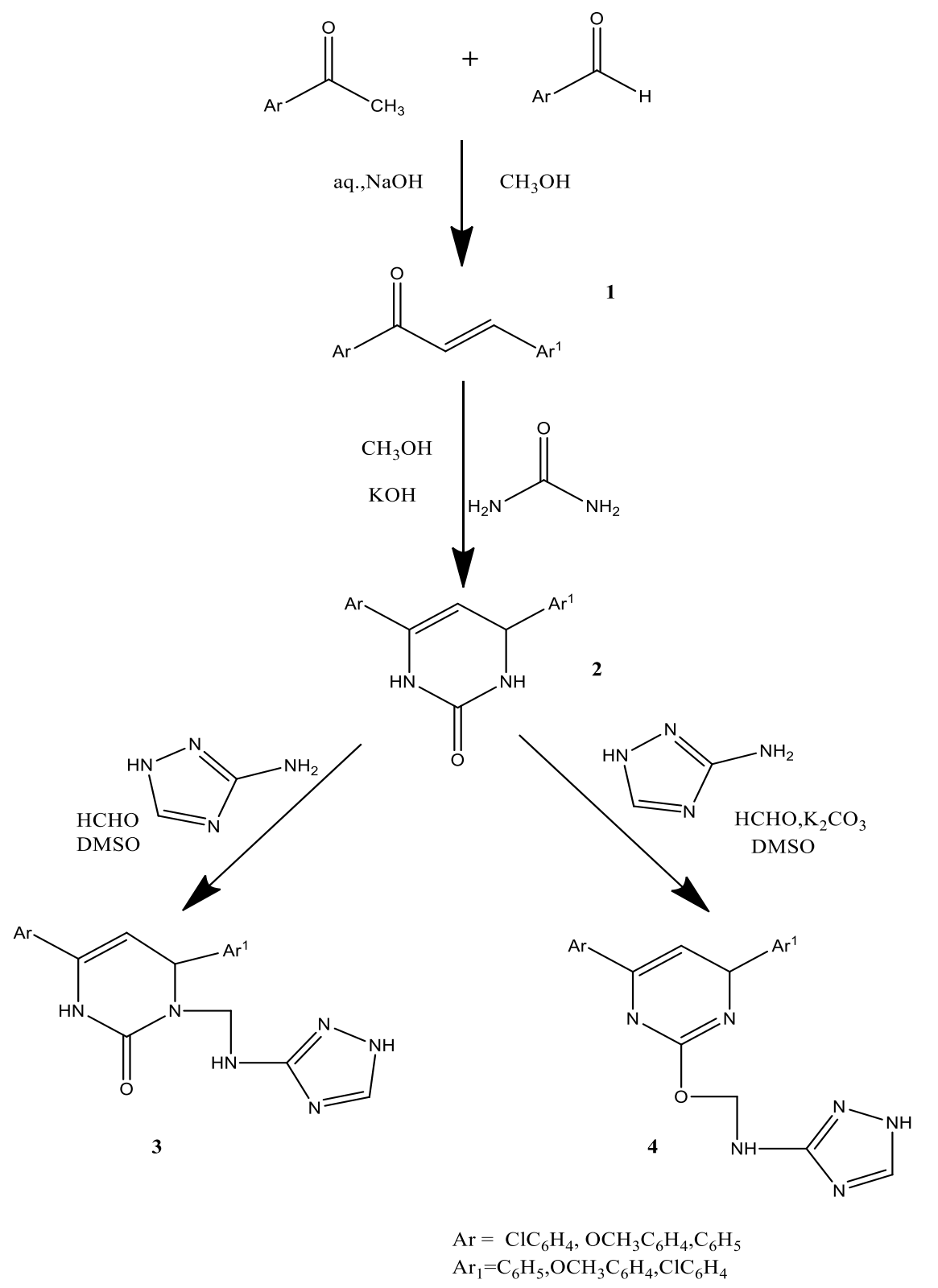

\section{Chemistry}

All the chemicals were purchased from Sd-Fine chemicals ltd, Ranbaxy chemicals ltd, Loba Chemicals ltd and Qualigens chemicals ltd. All the solvents used were of laboratory grade. All reactions were monitored by thin layer chromatography (TLC) on precoated silica gel 60 F254 (mesh); spots were visualized with UV light. Merck silica gel (100-200 mesh; Merck, Germany) was used for chromatography. All the synthesized compounds were purified by recrystallization (or) column chromatography. Melting points were determined on open capillary method and were uncorrected. IR spectra were recorded on a Perkin-Elmer FT-IR 240-C spectro photometer using $\mathrm{KBr}$ optics (Perkin-Elmer. USA). ${ }^{1} \mathrm{H}-\mathrm{NMR}$ spectra were recorded on Gemini Varian (Varian, USA) $200 \mathrm{MHz}$, Bruker (Bruker 
Bioscience, USA) AV $300 \mathrm{MHz}$, and Unity (Varian) $400 \mathrm{MHz}$ spectrometer in DMSO-d6 or $\mathrm{CDCl}_{3}$ using TMS as an internal standard.

\subsection{Synthesis of Chalcones}

A mixture of $22 \mathrm{gm}$ of $\mathrm{NaOH}$ in $200 \mathrm{ml}$ of water and $100 \mathrm{ml}$ of rectified spirit in a $500 \mathrm{ml}$ bolt head flask was provided with a magnetic stirrer. The flask was immersed in a bath of crushed ice, $0.43 \mathrm{~mol}$ of freshly distilled acetophenone was added while stirring and then 0.43 mole of pure benzaldehydes was added. The temperature of the mixture was kept at about $5^{\circ} \mathrm{C}$ (limits are $0-5^{\circ} \mathrm{C}$ ) and stirred vigorously until the mixture was so thick that stirring is no longer effective (2-3 h). The reaction was monitored by TLC. After completion of the reaction, stirrer was removed, and the reaction mixture was kept in ice chest or refrigerator overnight. Thus, obtained product was filtered and washed with cold water until the washings were neutral to litmus and then with $20 \mathrm{ml}$ of ice-cold rectified spirit and dried and re-crystallized from chloroform.

\subsection{Synthesis of Dihydropyrimidine -2-one (2)}

A mixture of 0.01 mole of chalcone, 0.01 mole of urea and $1 \mathrm{gm}$ of $\mathrm{KOH}$ were dissolved in minimum amount of ethanol in a glass beaker and were subjected to microwave irradiation at $80 \%$ power with the intervals of 10 seconds each. The reaction was monitored by TLC. After completion of the reaction, the contents were cooled to room temperature and poured into ice cold water $(50 \mathrm{ml})$ while stirring. The solid thus separated was filtered, washed with portions of cold water and dried. It was purified by recrystallization from ethanol to give a pure compound.

\subsection{Synthesis of N-Mannich Dihydropyrymidine Derivatives}

Dihydropyrimidine (2, 0.005 mole) was dissolved in minimum amount of DMSO in a glass beaker and 37\% HCHO $(0.01 \mathrm{~mole})$, and appropriate secondary amine $(0.005 \mathrm{~mol})$ was added. The reaction mixture was exposed to microwave irradiation at 480 micro power with the intervals of 1 minute each. The reaction progress was monitored by TLC and then kept at refrigerator for $48 \mathrm{~h}$, filtered the separated product, washed with small portions of cold water and dried. The crude product was purified by recrystallization from Pet. Ether: Chloroform (1:1 mixture).

\subsection{Synthesis of O-Mannich Dihydropyrimidine Derivatives}

Dihydropyrimidine (2, 0.005 mole) was dissolved in minimum amount of DMSO in a glass beaker and $37 \%$ HCHO (0.01 mole), anhydrous potassium carbonate $(1.0 \mathrm{~g})$, appropriate secondary amine $(0.005 \mathrm{~mol})$ was added and this reaction mixture was exposed to microwave irradiation at $80 \%$ power with the intervals of 20 seconds each. The reaction progress was monitored by TLC and the mixture was kept at refrigerator for $48 \mathrm{~h}$, filtered the separated product, washed with small portions of cold water and dried. The crude product was purified by recrystallization from Pet. Ether: Chloroform (1:1 mixture).

\section{Biological Activity}

\subsection{Cell Culture}

The human breast cancer (MCF-7 \&MDA MB231) cells were obtained from NCCS, Pune, India. Both cells lines were cultured in DMEM high glucose (Gibco, USA) supplemented 10\% fetal bivine serum (Gibco, USA) in a humidify incubator $37^{\circ} \mathrm{C}$ incubator containing $5 \% \quad \mathrm{CO}_{2}$ environment with $0.5 \%$ Penicillin-streptomycin (Sigma, USA). The medium was exchanged every 2 days with fresh medium to maintain cell activity.

\subsection{MTT Assay}

Breast cancers (MCF-7 \&MDA MB231) cells were plated in density of 5600 cells/100 $\mu 1$ and incubated in 96-well plate. The cells were exposed to various concentrations of dihydropyrimidine derivatives for 24 hours. Sensitivity of the agents were determined by the MTT assay (Promega, Mannheim, Germany) following the manufacturers' instructions in quadruplicates using a photometer at the wavelength of $490 \mathrm{~nm}$. $\mathrm{IC}_{50}$ values were determined from the growth inhibition curve.

\subsection{Cell Cycle Analysis}

Human breast cancers (MCF-7 \&MDA MB231) cells $\left(1 \times 10^{5}\right.$ cells/well) were seeded in 6-well plates. After $24 \mathrm{~h}$, the medium was replaced by fresh culture medium with IC50 concentrations of dihydropyrimidine derivatives (3a-f \&4a-f). The cell cycle assay was performed and analyzed by measuring the amount of propidium iodide (PI)-labeled DNA in ethanol-fixed cells. In brief, cells will be treated for $24 \mathrm{~h}$, harvested by trypsinization and fixed with chilled $70 \%$ ethanol. Cells were stained for total DNA content with PI $(5 \mathrm{mg} / \mathrm{mL})$ staining buffer has RNase enzyme according to the manufacturer's instructions. The percentages of cells in different phases Go-G1, S and G2-M were observed with flow cytometer, BD Aria II instrument and analyzed by Mod Fit software. 


\section{Result and Discussion}

The present study investigated anti-proliferative activity cell cycle distribution pattern of dihydropyrimidine derivatives in human breast cancer (MCF-7 \& MDA-MB-231) cell lines using in vitro model. It is important to select the appropriate concentrations for the treatment to find optimum concentrations. Here, we have treated cells with wide range of concentrations and optimized $\mathrm{IC}_{50}$ concentrations of dihydropyrimidine derivatives of $\mathbf{3 a - 3 f} \& \mathbf{4 a - 4 f}$ were fall in the range of 31.94-101.94 $\mu \mathrm{M}$ in MCF-7 cells as well as 32.06-98.04 in MDA-MB-231 cells (Table.1) of human breast cancer cell lines. Among all the compounds chloro and dichloro substitutions of $\mathbf{4 b}, \mathbf{4 e}$ and $\mathbf{3 e}$ were shown lesser $\mathrm{IC}_{50}$ concentration were $31.94,55.03$ and $55.73 \mu \mathrm{M}$ in MCF-7 cell line, whereas $41.50,32.06$ and $35.28 \mu \mathrm{M}$ in MDA-MB-231 cells. Here the both the cell lines were chemo sensitive to the dihydropyrimidine derivatives. But, triple negative and ER negative cell line of MDA-MB-231 were relatively more chemo sensitive than MCF-7 cells.

Table 1. The IC50 concentrations of dihydropyrimidine derivatives (3a-f \&4a-f) in human breast cancer (MCF-7\&MDA-MB 231) cell lines were estimated MTT assay

\begin{tabular}{l|c|c}
\hline Sample & MCF-7 Cells IC50 $\boldsymbol{\mu M}$ & MDA-MB 231 IC50 $\boldsymbol{\mu M}$ \\
\hline $3 \mathrm{a}$ & $104.94 \pm 0.47$ & $94.31 \pm 0.27$ \\
\hline $3 \mathrm{~b}$ & $72.94 \pm 0.22$ & $48.54 \pm 0.28$ \\
\hline $3 \mathrm{c}$ & $73.03 \pm 0.21$ & $85.05 \pm 0.21$ \\
\hline $3 \mathrm{~d}$ & $75.05 \pm 0.19$ & $73.94 \pm 0.18$ \\
\hline $\mathbf{3 e}$ & $\mathbf{5 5 . 7 3} \pm \mathbf{0 . 1 6}$ & $\mathbf{3 5 . 2 8} \pm \mathbf{0 . 1 9}$ \\
\hline $3 \mathrm{f}$ & $73.94 \pm 0.25$ & $40.62 \pm 0.19$ \\
\hline $4 \mathrm{a}$ & $95.04 \pm 0.48$ & $98.04 \pm 0.38$ \\
\hline $\mathbf{4 b}$ & $\mathbf{3 1 . 9 4} \pm \mathbf{0 . 1 2}$ & $\mathbf{4 1 . 5 0} \pm \mathbf{0 . 2 6}$ \\
\hline $4 \mathrm{c}$ & $80.64 \pm 0.34$ & $90.45 \pm 0.24$ \\
\hline $4 \mathrm{~d}$ & $79.39 \pm 0.22$ & $92.24 \pm 0.28$ \\
\hline $\mathbf{4 e}$ & $\mathbf{5 5 . 0 3} \pm \mathbf{0 . 2 7}$ & $\mathbf{3 2 . 0 6} \pm \mathbf{0 . 2 2}$ \\
\hline $4 \mathrm{f}$ & $73.51 \pm 0.34$ & $43.96 \pm 0.19$ \\
\hline Tamoxifen & $\mathbf{1 9 . 0 0} \pm \mathbf{0 . 1 6}$ & $\mathbf{2 3 . 4 6} \pm \mathbf{0 . 2 1}$ \\
\hline
\end{tabular}

However, these three selected compounds $(\mathbf{4 b}, \mathbf{4 e} \& \mathbf{3 e})$ were further studied their cell cycle regulation pattern in both breast cancer cells. Cell cycle progression of cell cycle regulation is a set of coordinated events happen in completion of one complete cell cycle. The cells distributed in to four major phases; G1 (growth phase 1), S (DNA synthesis phase), G2 (growth phase 2) and $\mathrm{M}$ (mitosis), which were functioning to integrate environment sensing signalling pathways with cell growth and proliferation (Ge'rard, C, et al., 2014). Most of anticancer drugs disturb proliferation cycle of tumour and inhibit the cell cycle events, which activate checkpoints, arrest cells and induce apoptosis (Chan, K.S, et al., 2012). Here, we have studied the cell cycle regulation pattern of selected compounds of $\mathbf{3 e}, \mathbf{4 e}$ and $\mathbf{4 b}$ dihydropyrimidines with DNA binding agent propidium iodide dye. The cells were arrested in s phase $\mathbf{3 e}, \mathbf{4 e} \& \mathbf{4} \mathbf{b}$ in MCF-7 cells (Figure.1A). Whereas $\mathbf{4 e}, \mathbf{4 b}$ arrested cells in $\mathrm{S}$ phase and $\mathbf{3 e}$ arrested cells in $\mathrm{G} 2-\mathrm{M}$ phase in MDA-MB-231 cell line indicated N-mannich base of chloro substitution compound arrested cells in proliferation stage (synthesis phase), whereas, $\mathbf{3 e}$ arrested cells in G2/M (mitotic phase) and $\mathbf{4 b}$ and $\mathbf{4 e}$ were arrested cells in S phase of cell cycle events in MDA-MB 231 cells (Figure.1B). Indicates the chloro and dichloro substitution in dihydropyrimidine derivatives were restricting cell cycle progression in human breast cancer cells, whereas the standard drug tamoxifen has been arrested cells in G0/G1 phase in both the cell lines. 

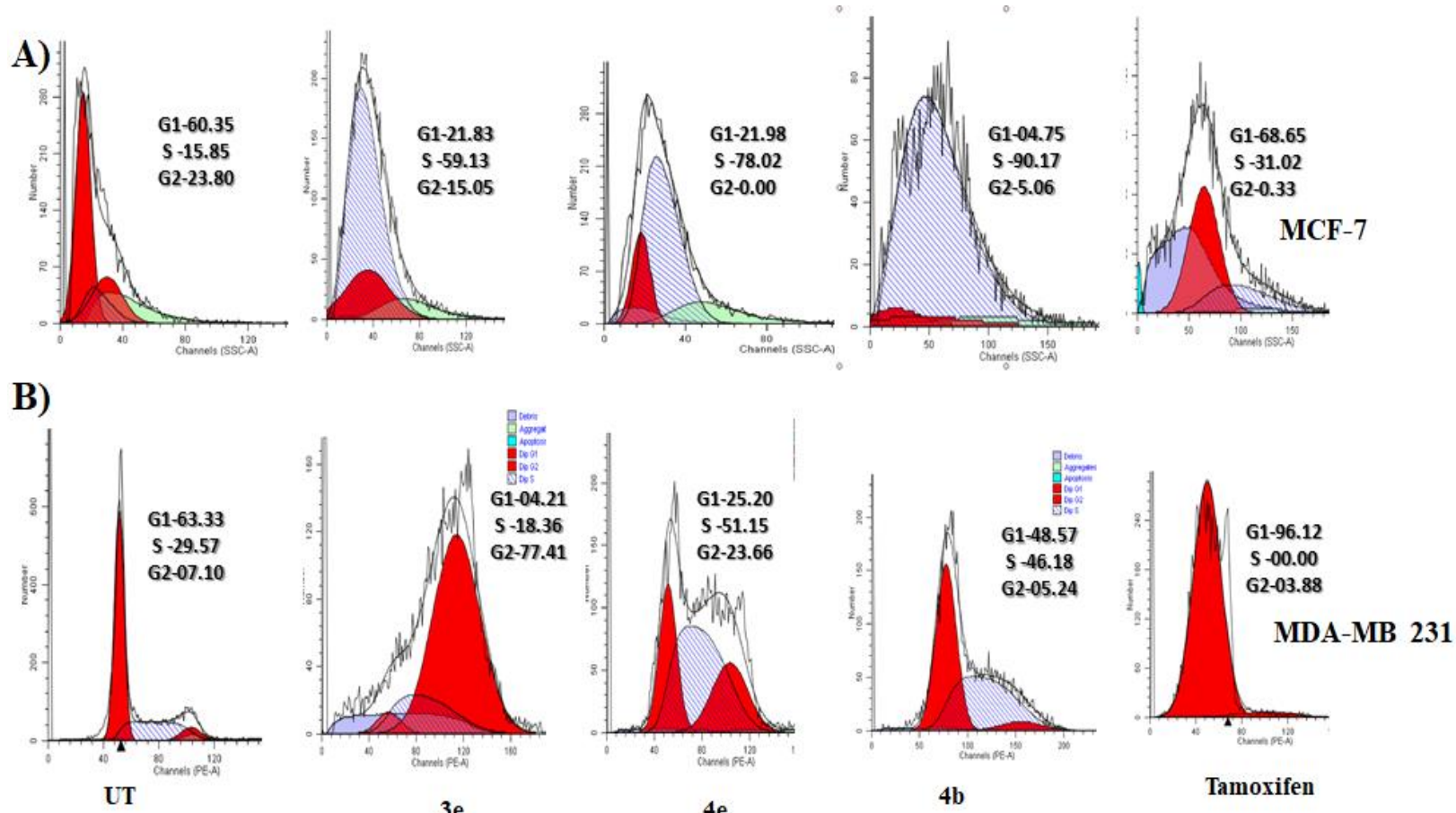

Figure 1. The cell cycle distribution pattern of dihydropyrimidine derivatives (3a-f \&4a-f) treatment in human breast cancer (MCF-7\&MDA-MB 231) cell lines were estimated flowcytometry

\section{Spectral Data of Dihydropyrimidine Derivatives}

5.1 4,6-Diphenyl-3-[(1H-[1,2,4] triazol-3-ylamino)-methyl]-3,4-dihydro-1H-pyrimidin-2-one (3a)

Yield: 43\%; mp: 190-192 ${ }^{\circ}$; IR (KBr disc, $\mathrm{Cm}^{-1}$ ): 3269 (N-H Stretch), 2924 (Ar-H), 1596, 1494 (Ar C=C Stretch), 1652 (C=O Stretch), 1214 (C-N stretch); ${ }^{1} \mathrm{H}$ NMR (400 MHz, DMSO-D6) $\delta 5.6$ (d,1H, pyrimidine $\mathrm{CH}$ ), 5.9 (d,1H,pyrimidine $\mathrm{C}=\mathrm{CH}), 8.9$ (t, H, C-NH-C, pyrimidine), $4.9\left(\mathrm{~d}, 2 \mathrm{H}, \mathrm{N}-\mathrm{CH}_{2}-\mathrm{N}\right), 5.2-5.3(\mathrm{~s}, 1 \mathrm{H}$, Pyrimidine $\mathrm{N}-\mathrm{H})$, 7.0-7.6 (m, 10H, Ar-H), $7.9(\mathrm{~d}, 1 \mathrm{H}$, triazole $\mathrm{CH}), 8.1(\mathrm{~d}, 1 \mathrm{H}$, triazole $\mathrm{NH})$; LC-MS: $m / z 247.0\left[\mathrm{M}^{+}+1\right]$.

\subsection{4-(4-Chloro-phenyl)-6-phenyl-3-[(1H-[1,2,4]triazol-3-ylamino)-methyl]-3,4-dihydro-1H-pyrimidin-2-one (3b)}

Yield: 45\%; mp: 315-8 ${ }^{\circ} \mathrm{C}$; IR (KBr disc, $\left.\mathrm{Cm}^{-1}\right)$ : 3258 (N-H Stretch), 2930 (Ar-H), 1591, 1488 (Ar C=C Stretch), 1649 (C=O Stretch), 1214 (C-N stretch), 1083 (C-Cl stretch); ${ }^{1} \mathrm{H}$ NMR (400 MHz, DMSO-D6) 5.65 (d,1H, pyrimidine $\mathrm{CH}$ ), $5.9(\mathrm{~d}, 1 \mathrm{H}$, pyrimidine $\mathrm{C}=\mathrm{CH}), 8.9(\mathrm{t}, \mathrm{H}, \mathrm{C}-\mathrm{NH}-\mathrm{C}$, pyrimidine $), 4.9\left(\mathrm{~d}, 2 \mathrm{H}, \mathrm{N}-\mathrm{CH}_{2}-\mathrm{N}\right), 5.2-5.3(\mathrm{~s}, 1 \mathrm{H}$, Pyrimidine $\mathrm{N}-\mathrm{H})$, 7.0-7.6 (m, 9H, Ar-H), $7.9(\mathrm{~d}, 1 \mathrm{H}$, triazole $\mathrm{CH}), 8.2(\mathrm{~d}, 1 \mathrm{H}$, triazole $\mathrm{NH})$; LC-MS: $m / z 382.0\left[\mathrm{M}^{+}+2\right]$.

\subsection{4-(4-Methoxy-phenyl)-6-phenyl-3-[(1H-[1,2,4]triazol-3-ylamino)-methyl]-3,4-dihy dro -1H-pyrimidin-2-one (3c)}

Yield: 42\%; mp: 335-8 ${ }^{\circ} \mathrm{C}$; IR (KBr disc, $\left.\mathrm{Cm}^{-1}\right): 3252$ (N-H Stretch), 2933 (Ar-H), 1589, 1487 (Ar C=C Stretch), 1654 (C=O Stretch), 1221 (C-N stretch), 1245, 1080 (C-O-C stretch); ${ }^{1} \mathrm{H}$ NMR (400 MHz, DMSO-D6) 3.8 (s, 3H, O-CH ( $_{3}$, $5.5(\mathrm{~d}, 1 \mathrm{H}$, pyrimidine $\mathrm{CH}), 5.8(\mathrm{~d}, 1 \mathrm{H}$, pyrimidine $\mathrm{C}=\mathrm{CH}), 8.9(\mathrm{t}, \mathrm{H}, \mathrm{C}-\mathrm{NH}-\mathrm{C}$, pyrimidine $), 4.9\left(\mathrm{~d}, 2 \mathrm{H}, \mathrm{N}-\mathrm{CH}_{2}-\mathrm{N}\right), 5.3(\mathrm{~s}$, $1 \mathrm{H}$, Pyrimidine N-H), 7.0-7.6 (m, 9H, Ar-H), $7.9(\mathrm{~d}, 1 \mathrm{H}$, triazole $\mathrm{CH}), 8.1(\mathrm{~d}, 1 \mathrm{H}$, triazole $-\mathrm{NH})$; LC-MS: $m / z 377.0\left[\mathrm{M}^{+}\right.$ $+1]$.

5.4 6-(4-Chloro-phenyl)-4-phenyl-3-[(1H-[1,2,4]triazol-3-ylamino)-methyl]-3,4-dihydro-1H-pyrimidin-2-one (3d)

Yield: 49\%; mp: 312-5 ${ }^{\circ} \mathrm{C}$; IR (KBr disc, $\mathrm{Cm}^{-1}$ ): 3248 (N-H Stretch), 2920 (Ar-H), 1577, 1458 (Ar C=C Stretch), 1639 (C=O Stretch), 1234 (C-N stretch), 1075 (C-Cl stretch); ${ }^{1} \mathrm{H}$ NMR (400 MHz, DMSO-D6) 5.6 (d,1H, pyrimidine $\mathrm{CH}$ ), $5.9(\mathrm{~d}, 1 \mathrm{H}$,pyrimidine $\mathrm{C}=\mathrm{CH}), 8.9\left(\mathrm{t}, \mathrm{H}, \mathrm{C}-\mathrm{NH}-\mathrm{C}\right.$, pyrimidine), $4.9\left(\mathrm{~d}, 2 \mathrm{H}, \mathrm{N}-\mathrm{CH}_{2}-\mathrm{N}\right), 5.2-5.3(\mathrm{~s}, 1 \mathrm{H}$, Pyrimidine $\mathrm{N}-\mathrm{H})$, 7.0-7.6 (m, 9H, Ar-H), $7.9(\mathrm{~d}, 1 \mathrm{H}$, triazole $\mathrm{CH}), 8.1(\mathrm{~d}, 1 \mathrm{H}$, triazole $\mathrm{NH})$; LC-MS: $m / z 382.0\left[\mathrm{M}^{+}+2\right]$.

5.5 4,6-Bis-(4-chloro-phenyl)-3-[(1H-[1,2,4]triazol-3-ylamino)-methyl]-3,4-dihydro-1H-pyrimidin-2-one (3e)

Yield: 48\%; mp: 320-2 ${ }^{\circ} \mathrm{C}$; IR (KBr disc, $\mathrm{Cm}^{-1}$ ): 3238 (N-H Stretch), 2914 (Ar-H), 1566, 1448 (Ar C=C Stretch), 1633 (C=O Stretch), 1230 (C-N stretch), 1085, 1106 (C-Cl stretch); ${ }^{1} \mathrm{H}$ NMR (400 MHz, DMSO-D6) 5.4 (d,1H, pyrimidine $\mathrm{CH}), 5.8(\mathrm{~d}, 1 \mathrm{H}$,pyrimidine $\mathrm{C}=\mathrm{CH}), 8.9\left(\mathrm{t}, \mathrm{H}, \mathrm{C}-\mathrm{NH}-\mathrm{C}\right.$, pyrimidine), $4.9\left(\mathrm{~d}, 2 \mathrm{H}, \mathrm{N}-\mathrm{CH}_{2}-\mathrm{N}\right), 5.2-5.3(\mathrm{~s}, 1 \mathrm{H}$, Pyrimidine $\mathrm{N}-\mathrm{H})$, 7.0-7.6 (m, 9H, Ar-H), $7.9(\mathrm{~d}, 1 \mathrm{H}$, triazole $\mathrm{CH}), 8.3(\mathrm{~d}, 1 \mathrm{H}$, triazole $\mathrm{NH})$; LC-MS: $m / z 416.0\left[\mathrm{M}^{+}+2\right]$. 
5.6 6-(4-Chloro-phenyl)-4-(4-methoxy-phenyl)-3-[(1H-[1,2,4]triazol-3-ylamino)-methyl]-3,4-dihydro-1H-pyrimidin-2-o $n e(3 f)$

Yield: 45\%; mp: 338-40 C; IR (KBr disc, $\mathrm{Cm}^{-1}$ ): 3257 (N-H Stretch), 2933 (Ar-H), 1542, 1455 (Ar C=C Stretch), 1650 (C=O Stretch), 1227 (C-N stretch), 1102 (C-Cl stretch), 1068 (C-O-C stretch); ${ }^{1} \mathrm{H}$ NMR (400 MHz, DMSO-D6) 5.4 $(\mathrm{d}, 1 \mathrm{H}$, pyrimidine $\mathrm{CH}), 5.9(\mathrm{~d}, 1 \mathrm{H}$,pyrimidine $\mathrm{C}=\mathrm{CH}), 8.9(\mathrm{t}, \mathrm{H}, \mathrm{C}-\mathrm{NH}-\mathrm{C}$, pyrimidine $), 4.9\left(\mathrm{~d}, 2 \mathrm{H}, \mathrm{N}-\mathrm{CH}_{2}-\mathrm{N}\right), 5.2(\mathrm{~s}, 1 \mathrm{H}$, Pyrimidine N-H), 7.0-7.6 (m, 9H, Ar-H), 7.9 (d,1H, triazole $\mathrm{CH}), 8.2(\mathrm{~d}, 1 \mathrm{H}$, triazole $\mathrm{NH})$; LC-MS: $\mathrm{m} / z .412 .0\left[\mathrm{M}^{+}+2\right]$.

$5.7 \mathrm{~N}$-\{[(4,6-diphenyl-1,2,3,4-tetrahydropyrimidin-2-yl)oxy]methyl\}-1H-1,2,4-triazol-3-amine (4a):

Yield: 45\%; mp: 315-7 ${ }^{\circ} \mathrm{C}$; IR (KBr disc, $\mathrm{Cm}^{-1}$ ): 3057 (N-H Stretch), 2848 (Ar-H), 1576 (Aromatic C=C Stretch), 1662 (C-O Stretch), 3025 (Ar-H). 1218 (C-N Stretch); ${ }^{1} \mathrm{H}$ NMR (400 MHz, DMSO-D6) 4.9 (d,1H, pyrimidine CH), 5.9 (d,1H,pyrimidine $\mathrm{C}=\mathrm{CH}), 8.9$ (t, H, C-NH-C, pyrimidine), $4.9\left(\mathrm{~d}, 2 \mathrm{H}, \mathrm{N}-\mathrm{CH}_{2}-\mathrm{N}\right), 5.2-5.3(\mathrm{~s}, 1 \mathrm{H}$, Pyrimidine $\mathrm{N}-\mathrm{H})$, 6.8-7.6 $(\mathrm{m}, 10 \mathrm{H}, \mathrm{Ar}-\mathrm{H}), 7.9(\mathrm{~d}, 1 \mathrm{H}$, triazole $\mathrm{CH}), 8.1(\mathrm{~d}, 1 \mathrm{H}$, triazole $\mathrm{NH})$; LC-MS: $m / z, 347.0\left[\mathrm{M}^{+}+1\right]$.

\section{$5.8 \mathrm{~N}$-(\{[4-(4-chlorophenyl)-6-phenyl-1,2,3,4-tetrahydropyrimidin-2-yl]oxy\}methyl)-1H-1,2,4-triazol-3-amine (4b)}

Yield: 49\%; mp: 310-12 ${ }^{\circ} \mathrm{C}$; IR (KBr disc, $\mathrm{Cm}^{-1}$ ): 3052 (N-H Stretch), 2850 (Ar-H), 1569 (Aromatic C=C Stretch), 1666 (C-O Stretch), 3035 (Ar-H). 1222 (C-N Stretch), 1099 (C-Cl stretch); ${ }^{1} \mathrm{H}$ NMR (400 MHz, DMSO-D6) $\delta 4.9$ (d,1H, pyrimidine $\mathrm{CH}), 5.9(\mathrm{~d}, 1 \mathrm{H}$, pyrimidine $\mathrm{C}=\mathrm{CH}), 8.9\left(\mathrm{t}, \mathrm{H}, \mathrm{C}-\mathrm{NH}-\mathrm{C}\right.$, pyrimidine), $4.9\left(\mathrm{~d}, 2 \mathrm{H}, \mathrm{N}_{-} \mathrm{CH}_{2}-\mathrm{N}\right), 5.2-5.3(\mathrm{~s}, 1 \mathrm{H}$, Pyrimidine N-H), 7.0-7.6 (m, 5H, Ar-H), 7.5-7.6 (m, 2H, Ar-H), 7.4-7.5 (m,2H,Ar-H), 7.8-7.9 (d,1H, triazole $\mathrm{CH})$, 7.9-8.0 (d,1H,triazole NH); LC-MS: $m / z, 382.0\left[\mathrm{M}^{+}+2\right]$.

5.9 [4-(4-Methoxy-phenyl)-6-phenyl-1,2,3,4-tetrahydro-pyrimidin-2-yloxymethyl]-(1H-[1,2,4]triazol-3-yl)-amine (4c) $\mathrm{C}_{20} \mathrm{H}_{20} \mathrm{~N}_{6} \mathrm{O}_{2}$

Yield: 42\%; mp: 325-7 ${ }^{\circ} \mathrm{C}$; IR (KBr disc, $\mathrm{Cm}^{-1}$ ): 3044 (N-H Stretch), 2839 (Ar-H), 1567 (Aromatic C=C Stretch), 1658 (C-O Stretch), 3055 (Ar-H), 1228 (C-N Stretch), 1252, 1100 (C-O-C stretch); ${ }^{1}$ H NMR (400 MHz, DMSO-D6) 4.9 $(\mathrm{d}, 1 \mathrm{H}$, pyrimidine $\mathrm{CH}), 5.9(\mathrm{~d}, 1 \mathrm{H}$, pyrimidine $\mathrm{C}=\mathrm{CH}), 8.9(\mathrm{t}, \mathrm{H}, \mathrm{C}-\mathrm{NH}-\mathrm{C}$, pyrimidine $), 4.9\left(\mathrm{~d}, 2 \mathrm{H}, \mathrm{N}-\mathrm{CH}_{2}-\mathrm{N}\right), 5.2-5.3(\mathrm{~s}$, $1 \mathrm{H}$, Pyrimidine $\mathrm{N}-\mathrm{H}), 7.0-7.6(\mathrm{~m}, 9 \mathrm{H}, \mathrm{Ar}-\mathrm{H}), 3.4-3.5\left(\mathrm{~s}, 3 \mathrm{H}, \mathrm{OCH}_{3}\right), 7.8-7.9(\mathrm{~d}, 1 \mathrm{H}$, triazole $\mathrm{CH}), 7.9-8.0(\mathrm{~d}, 1 \mathrm{H}$, triazole NH); LC-MS: $m / z, 377.0\left[\mathrm{M}^{+}+1\right]$.

$5.10 \mathrm{~N}-(\{[6-(4-c h l o r o p h e n y l)-4-p h e n y l-1,2,3,4$-tetrahydropyrimidin-2-yl]oxy\}methyl)-1H 1,2,4-triazol-3-amine (4d) $\mathrm{C}_{19} \mathrm{H}_{17} \mathrm{ClN}_{6} \mathrm{O}$ :

Yield: 52\%; mp: 310-2 ${ }^{\circ} \mathrm{C}$; IR (KBr disc, $\left.\mathrm{Cm}^{-1}\right): 3248$ (N-H Stretch), 2920 (Ar-H), 1577, 1458 (Ar C=C Stretch), 1639 (C-O Stretch), 1234 (C-N stretch), 3055 (Ar-H), 1070 (C-Cl stretch); ${ }^{1} \mathrm{H}$ NMR (400 MHz, DMSO-D6) $\delta 5.8$ (d,1H pyrimidine $\mathrm{CH}-\mathrm{Ar}) 3.7(\mathrm{~d}, 1 \mathrm{H}$,pyrimidine, $\mathrm{C}=\mathrm{CH}) 4.8(\mathrm{t}, 1 \mathrm{H}, \mathrm{C}-\mathrm{NH}-\mathrm{C}$ triazole $), 5.3\left(\mathrm{~d}, 2 \mathrm{H}, \mathrm{O}-\mathrm{CH}_{2}-\mathrm{N}\right), 3.4(\mathrm{~s}, 1 \mathrm{H}$, Pyrimidine N-H), 7.0-7.6 (m, 5H, Ar-H), $7.8(\mathrm{~m}, 2 \mathrm{H}, \mathrm{Ar}-\mathrm{H}), 7.9(\mathrm{~m}, 2 \mathrm{H}, \mathrm{Ar}-\mathrm{H}), 7.2(\mathrm{~d}, 1 \mathrm{H}$, triazole $\mathrm{CH}), 7.9-8.0$ (d,1H,triazole -NH); LC-MS: $\mathrm{m} / 2,382.0\left[\mathrm{M}^{+}+2\right]$.

5.11 [4,6-Bis-(4-chloro-phenyl)-1,2,3,4-tetrahydro-pyrimidin-2-yloxymethyl]-(1H-[1,2,4] triazol-3-yl)-amine (4e) $\mathrm{C}_{19} \mathrm{H}_{16} \mathrm{Cl}_{2} \mathrm{~N}_{6} \mathrm{O}$

Yield:46\%; mp: 318-20 ${ }^{\circ} \mathrm{C}$; IR (KBr disc, $\mathrm{Cm}^{-1}$ ): 3044 (N-H Stretch), 2839 (Ar-H), 1567 (Aromatic C=C Stretch), 1658 (C-O Stretch), 3055 (Ar-H), 1228 (C-N Stretch), 1086 (C-Cl stretch); ${ }^{1} \mathrm{H}$ NMR (400 MHz, DMSO-D6) $\delta 5.7$ (d,1H pyrimidine $\mathrm{CH}), 5.4(\mathrm{~d}, 1 \mathrm{H}$, pyrimidine, $\mathrm{C}=\mathrm{CH}) 4.9(\mathrm{t}, 1 \mathrm{H}, \mathrm{C}-\mathrm{NH}-\mathrm{C}), 5.3\left(\mathrm{~d}, 2 \mathrm{H}, \mathrm{O}-\mathrm{CH}_{2}-\mathrm{N}\right), 5.2(\mathrm{~s}, 1 \mathrm{H}$, Pyrimidine $\mathrm{N}-\mathrm{H})$, 7.0-7.6 (m, 8H, Ar-H), $7.9(\mathrm{~d}, 1 \mathrm{H}$, triazole $\mathrm{CH}), 8.2\left(\mathrm{~d}, 1 \mathrm{H}\right.$, triazole NH); LC-MS: $m / z 416.0\left[\mathrm{M}^{+}+2\right]$.

5.12 [6-(4-Chloro-phenyl)-4-(4-methoxy-phenyl)-1,2,3,4-tetrahydro-pyrimidin-2-yloxymethyl]-(1H-[1,2,4] triazol-3-yl)-amine (4f) $\mathrm{C}_{20} \mathrm{H}_{19} \mathrm{ClN}_{6} \mathrm{O}_{2}$

Yield: 45\%; mp: 328-30 ${ }^{\circ} \mathrm{C}$; IR (KBr disc, $\mathrm{Cm}^{-1}$ ): 3034 (N-H Stretch), 2842 (Ar-H), 1568 (Aromatic C=C Stretch), 1659 (C-O Stretch), 3048 (Ar-H), 1232 (C-N Stretch), 1245, 1095 (C-O-C stretch), 1083 (C-Cl stretch); ${ }^{1} \mathrm{H}$ NMR (400 MHz, DMSO-D6) $\delta 5.3(\mathrm{~d}, 1 \mathrm{H}$ pyrimidine $\mathrm{CH}-\mathrm{Ar}) 4.9(\mathrm{~d}, 1 \mathrm{H}$,pyrimidine, $\mathrm{C}=\mathrm{CH}) 5.3\left(3 \mathrm{H}, \mathrm{OCH}_{3} 4.8-4.9(\mathrm{t}, \mathrm{H}, \mathrm{C}-\mathrm{NH}-\mathrm{C}\right.$, triazole), $5.1\left(\mathrm{~d}, 2 \mathrm{H}, \mathrm{O}-\mathrm{CH}_{2}-\mathrm{N}\right), 4.8(\mathrm{~s}, 1 \mathrm{H}$, Pyrimidine $\mathrm{N}-\mathrm{H}), 7.0-7.6(\mathrm{~m}, 8 \mathrm{H}, \mathrm{Ar}-\mathrm{H}), 7.8-7.9(\mathrm{~d}, 1 \mathrm{H}$, triazole $\mathrm{CH}), 7.9-8.0$ (d,1H,triazole NH); LC-MS: $m / z 412.0\left[\mathrm{M}^{+}+2\right]$. 
NMR Spectra: 3a

$\mathbf{z}$
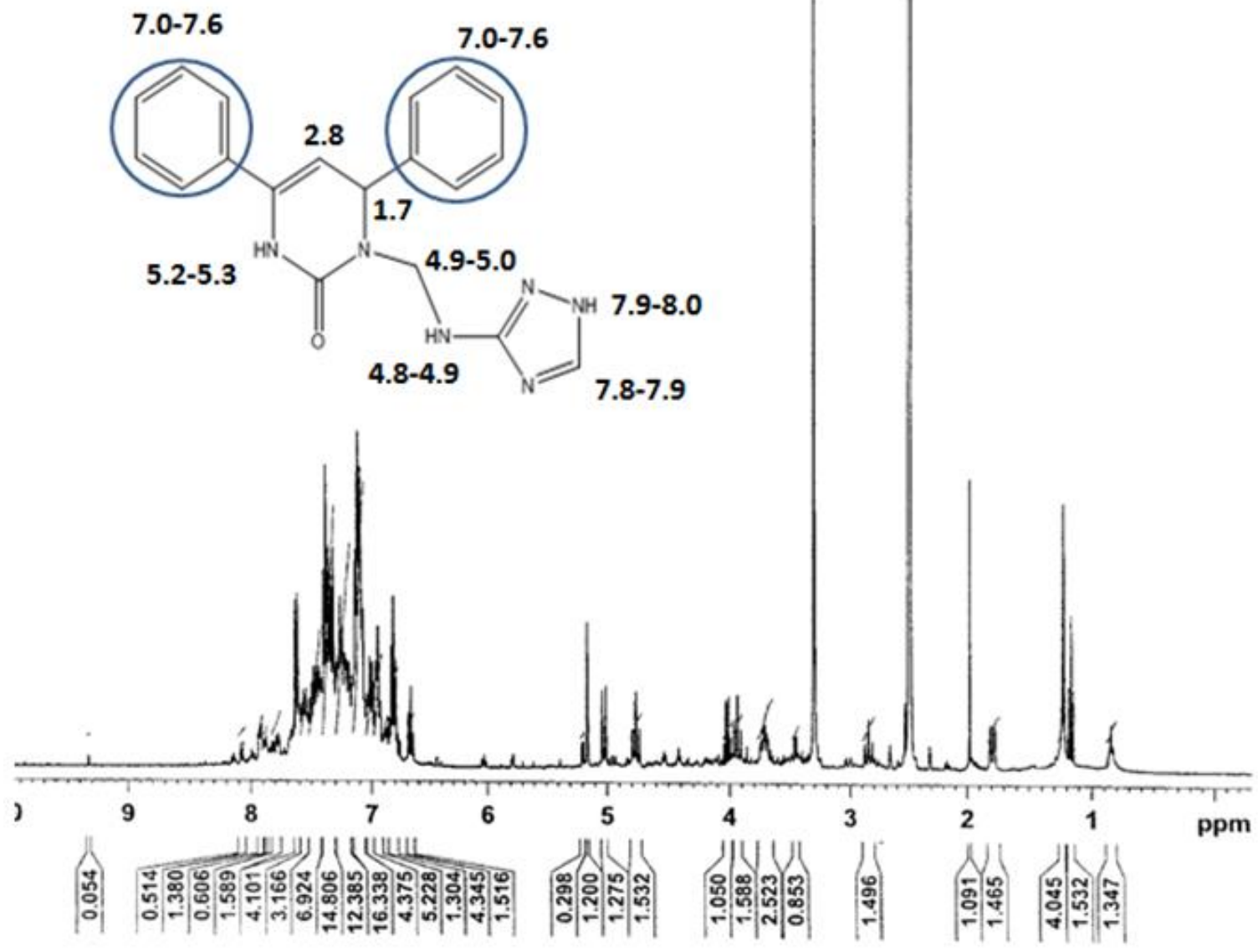
NMR Spectra: 4a

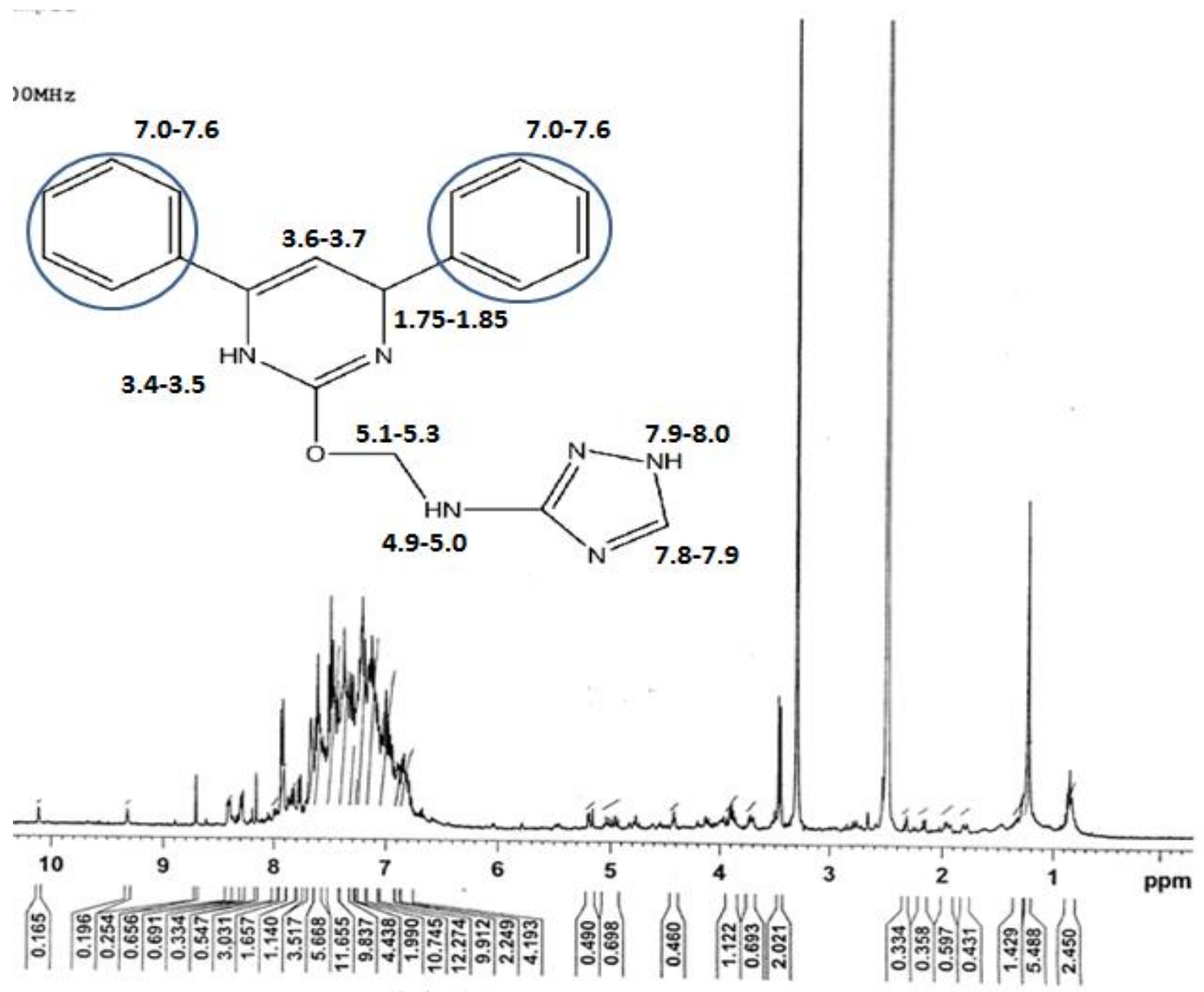

\section{Conclusion}

In the present work, new dihydropyrimidine derivatives of $\mathrm{N}(\mathbf{3 a - 3})$ and $\mathrm{O}(\mathbf{4 a - 4 f})$ - mannich derivatives of dihydropyrimidine were designed, synthesised, characterized and investigated for their inhibitory activity and cell cycle analysis in human breast cancer (MCF-7\&MDA-MB-231) cell lines. Among these compounds $3 \mathrm{c}$ and $4 \mathrm{~b}$ found with cell cycle arrest in G2-M phase. The apoptosis assay also revealed the compounds of chloro substitution (3c \& $\mathbf{4 b}$ ) were having more potent with $74 \%$ inhibition. These were comparable with standard drug tamoxifen.

\section{Acknowledgements}

AR acknowledges for support of grants ICMR-NIN, Indian Council of Medical Research [ICMR], Government of India. SB and VV are Co-Investigators of the projects. SB has helped in synthesis of dihydropyrimidine derivatives, GT and $\mathrm{BP}$ are assisted in carrying the work.

\section{References}

Ajmal, R. B. (2017). Biological activity of pyrimidine derivatives: A review. Organic \& Medicinal Chem.I. J., 2(2), OMCIJ.MS.ID.555-581.

Ajmal, R. B., Rajendra, S. D., Gowhar, A. N., Israr, U. H., \& Tabassum, A. (2017). Proficient synthesis of bioactive annulated pyrimidine derivatives: A review, Journal of Taibha University for Science, 11(6), 1047-1069. https://doi.org/10.1016/j.jtusci.2017.05.005

Bandgar, B. P., Gawande, S. S., Bodade, R. G., Totre, J. V., \& Khobragade C. N. (2010). Synthesis and biological evaluation of simple methoxylated chalcones as anticancer, anti-inflammatory and antioxidant agents. Bioorg.. Med. Chem., 18(3), 1364-1370. https://doi.org/10.1016/j.bmc.2009.11.066

Birendra, N. G., Jiban, C. S. K., \& Jogendra, N. B. (1984). Synthesis and antibacterial activity of 1-(2,4-dichlorobenzoyl)-4-substituted thiosemicarbazides, 1,2,4-triazoles and their methyl derivatives, $J$. Heterocycl. Chem., 21, 12-25. https://doi.org/10.1002/jhet.5570210460 
Breault, G. A., Baettie, J. F., Ellston, P. A., Jewsbury, P. J, Midgley, C. J., Naven, R. T., ... \& Tucker, J. A. (2003). Cyclin-dependent kinase 4 inhibitors as a treatment for cancer. Part 1: identification and optimisation of substituted 4, 6-bis anilino pyrimidines. Bioorg. Med. Chem. Lett.. 13(14), 2955-2961. https://doi.org/10.1016/S0960-894X(03)00203-8

Bruno, O., Schenone, S., Ranise, A., Bondavalli, F., Barocelli, E.,... \& Impicciatore, M. (2001). New polycyclic pyrimidine derivatives with antiplatelet in vitro activity: synthesis and pharmacological screening. Bioorg. Med. Chem., 9(3), 629-636. https://doi.org/10.1016/S0968-0896(00)00272-8

Cernecka, H., Veizerova, L., Mensikova, L., Svetlik, J., \& Krenek, P. (2012). Selective inhibitory action of Biginelli-type dihydropyrimidines on depolarization-induced arterial smooth muscle contraction. J. Pharm. Pharmacol., 64(5), 735-741. https://doi.org/10.1111/j.2042-7158.2012.01466.x

Chan, K. S., Koh, C. G., \& Li, H. Y. (2012). Mitosis-targeted anti-cancer therapies: where they stand. Citation: Cell Death and Disease, 3, e411. https://doi.org/10.1038/cddis.2012.148

Chikhale, R. V., Bhole, R. P., Khedekar, P. B., \& Bhusari, K. P. (2009). Synthesis and pharmacological investigation of 3-(substituted 1-phenylethanone)-4-(substituted phenyl)-1, 2, 3, 4-tetrahydropyrimidine-5-carboxylates. Eur. J. Med. Chem., 44(9), 3645-3653. https://doi.org/10.1016/j.ejmech.2009.02.021

Dobrusin, E. M., Hamby, J. M., Kramer J. B., Schroeder, M. C., Showalter, H. D. H., Toogood, P., \& Susanne, A. T. (2001). Bicyclic pyrimidines and bicyclic 3,4-dihydropyrimidines as inhibitors of cellular proliferation. Patent, EP $1080092 \mathrm{~A} 2$.

Ganapathi, T., Rajanna, A., \& Vijayalakshmi, V. (2017). Novel dihydropyrimidine derivatives as potential HDAC inhibitors: in silico study. In Silico Pharmacol, 5, 10, https://doi.org/10.1007/s40203-017-0030-4

Ge'rard, C., \& Goldbeter, A. (2014). The balance between cell cycle arrest and cell proliferation: control by the extracellular matrix and by contact inhibition. Interface Focus, 4. https://doi.org/10.1098/rsfs.2013.0075

Haque, S. A., Hasaka, T. P., Brooks, A. D., Lobanov, P. V., \& Baas P. W. (2004). Monastrol, a prototype anti-cancer drug that inhibits a mitotic kinesin, induces rapid bursts of axonal outgrowth from cultured postmitotic neurons. Cell Motil. Cytoskeleton, 58(1), 10-16. https://doi.org/10.1002/cm.10176

Harun, M. P., Malleshappa, N. N., Navdeep, S. S., Andanappa, K. G., \& Swaranjit, S. C. (2017). Synthesis and antitubercular evaluation of imidazo[2,1-b][1,3,4]thiadiazole derivatives, Arabian Journal of Chemistry, 10, S996-S1002. https://doi.org/10.1016/j.arabjc.2013.01.001

Hockova, D., Holy, A., Snoeck, R., \& Clercq, E. D. (2003). 5-Substituted-2, 4-diamino-6-[2-(phosphonomethoxy) ethoxy]pyrimidines-acyclic nucleoside phosphonate analogues with antiviral activity. J. Med. Chem., 46, 5064-5073. https://doi.org/10.1021/jm030932o

Koksal, M., Okhan, N. G., Upeli, E. K., Yesilada, E., \& Erdogan, H. (2007). Analgesic and antiinflmmatory activities of some new Mannich bases of 5-nitro-2-benzoxazolinones. Archives of Pharmacal Research, 30(4), 419-424. https://doi.org/10.1007/BF02980214

Kothari, P. J., Mehlhoff, M. A., Singh, S. P., Parmar, S. S., and Stenberg, V. I. (1980). Synthesis of 5-(1-naphthylmethyl)-4-aryl-s-triazol-3-thiols/ylthioglycolic acids as possible antiinflammatory agents, $J$. Heterocycl. Chem., 17, 1369. https://doi.org/10.1002/jhet.5570170703

Kothari, P. J., Singh, S. P., Parmar, S. S., and Stenberg, V. I. (1980). Synthesis of some newer 5-(5-aryl-2H-tetrazol-2-ylmethyl)-4-substituted-s-triazole-3-thiols as possible antiinflammatory agents, $J$. Heterocycl. Chem., 17, 1393. https://doi.org/10.1002/jhet.5570170709

Lauro, F. V., Francisco, D. C., Maria, L. R., \& Elodia, G. C. (2010). Activity induced by two Steroid-Dihydropyrimidine derivatives on Glucose levels in a Diabetic Rat Model. Relationship between descriptors $\log \mathrm{P}$ and its Antidiabetic activity. Inter J of PharmTech Research, 2(3), 2075-2080.

Pandeya, S. N., Sriram, D., Nath, G., \& De Clercq, E. (2000). Synthesis, antibacterial, antifungal and anti-HIV activities of norfloxacin Mannich bases. Eur. J. Med. Chem., 35(2), 249-255.

https://doi.org/10.1016/S0223-5234(00)00125-2

Rami, C., Patel, L., Patel, C. N., \& Parmar, J. P. (2013). Synthesis, antifungal activity, and QSAR studies of 1,6-dihydropyrimidine derivatives. J Pharm Bioallied Sci., 5(4), 277-289.

https://doi.org/10.4103/0975-7406.120078 
Sangaraiah, N., Murugan, S., Poovan, S., Raja, R., Alagusundaram, P., Ramakrishnan, V., \& Vellasamy, S. (2012). Facile water promoted synthesis of 1, 2, 3-triazolyl dihydropyrimidine-2-thione hybrids-highly potent antibacterial agents. Eur J Med Chem., 58, 464-469. https://doi.org/10.1016/j.ejmech.2012.10.029

Sarmah, S. C., and Bahel, S. C. (1982). Synthesis and antimicrobial activity of 1,2,3-triazoles containing quinoline moiety. J. Indian Chem. Soc., 59, 877.

Schneider, P., Hawser, S., \& Islam, K. (2003). Iclaprim, a novel diaminopyrimidine with potent activity on trimethoprim sensitive and resistant bacteria. Bioorg. Med. Chem. Lett. 13(23), 4217-4221. https://doi.org/10.1016/j.bmcl.2003.07.023

Sengupta, A. K., and Misra, H. K. (1981). Synthesis of 1,2,4-triazole derivatives containing benzothiazoles as pharmacologically active molecule. J. Indian Chem. Soc., 58, 508.

Shah, T. B., Gupte, A., Patel, M. R., Chaudari, V. S., Patel, H., \& Patel, V. C. (2010). Synthesis and in vitro study of biological activity of heterocyclic N-Mannich bases of 3, 4-dihydropyrimidune-2 (1H)-thiones. Indian J. Chem, 49B, 578-786.

Sosnicki, J. G., Struk, L., Kurzawski, M., Peruzynska, M., Maciejewska, G., \& Drozdzik, M. ( 2014). Regioselective synthesis of novel 4,5-diaryl functionalized 3,4-dihydropyrimidine-2(1H)-thiones via a non-Biginelli-type approach and evaluation of their in vitro anticancer activity. Org Biomol Chem., 12(21), 3427-3440.

Sriram, D., Banerjee, D., \& Yogeeswari, P. (2009). Efavirenz Mannich bases: synthesis, anti-HIV and antitubercular activities. J Enzy Inhibition Med Chem, 24(1), 1-5. https://doi.org/10.1080/14756360701404159

Trivedi, A., Dodiya, D., Surani, J., Jarsania, S., Mathukiya, H.,... \& Shah, V. (2008). Facile one-pot synthesis and antimycobacterial evaluation of pyrazolo[3,4-d]pyrimidines. Arch Pharm (Weinheim), 341(7), 435-439. https://doi.org/10.1002/ardp.200800027

Vashishtha, S. C., Zello, G. A., Nienaber, K. H., Balzarini, J., De Clercq, E., Stables, J. P., ... \& Dimmock, J. R. (2004). Cytotoxic and anticonvulsant aryloxyaryl Mannich bases and related compounds. Eur. J. of Med. Chem., 39(1) 27-35. https://doi.org/10.1016/j.ejmech.2003.09.011

\section{Copyrights}

Copyright for this article is retained by the author(s), with first publication rights granted to the journal.

This is an open-access article distributed under the terms and conditions of the Creative Commons Attribution license (http://creativecommons.org/licenses/by/4.0/). 